\title{
Géneros Literarios en la Destreza Lectora del Idioma Inglés
}

\section{Literary Genres in the Reading Skill of the English Language}

\author{
Evelyn Vanessa Rodríguez Salinas. ${ }^{1}$, Ana Jazmina Vera de la Torre. ${ }^{2}$, Elsa Mayorie \\ Chimbo Cáceres. ${ }^{3}$, Wilma Elizabeth Suárez Mosquera. ${ }^{4}$
}

\begin{abstract}
This article engages the advantages of the proper use of literary genres in the reading skill of the English language. Due to the fact, Ecuadorian people are not frequent readers, it is necessary to create the reader habit in the Education System of this South American country. The best manner to introduce a reading habit in the curricula is to include activities that involve the use of literary Genres in the learning of English. In view of the mentioned purpose, a qualitative-quantitative methodology was applied through a structured survey conducted to the students and the teacher of the sixth and seventh year of an Elementary Education School. The results obtained were analyzed through the Chisquare statistical test, the same which points out that the proper use of literary genres in activities related to the development of the reading skill of the English language has achieved great advantages.
\end{abstract}

Keywords: Literary Genres, Reading Skill, English Language, great advantages.

\begin{abstract}
Abstrac.
Este artículo aborda las ventajas del uso adecuado de los géneros literarios en la habilidad de lectura del idioma inglés. Debido al hecho de que los ecuatorianos no son lectores frecuentes, es necesario crear el hábito del lector en el Sistema Educativo de este país sudamericano. La mejor manera de introducir un hábito de lectura en los planes de estudio es incluir actividades que involucren el uso de géneros literarios en el aprendizaje del inglés. En vista del propósito mencionado, se aplicó una metodología cualitativacuantitativa a través de una encuesta estructurada realizada a los estudiantes y al maestro

\footnotetext{
${ }^{1}$ Universidad Técnica de Ambato. vaners.vaners@ gmail.com

${ }^{2}$ Universidad Técnica de Ambato. Docente de la Carrera de Pedagogía de los Idiomas Nacionales y Extranjeros. aj.vera@uta.edu.ec

${ }^{3}$ Universidad Técnica de Ambato. Docente de la Carrera de Pedagogía de los Idiomas Nacionales y Extranjeros. elsamchimboc@uta.edu.ec

${ }^{4}$ Universidad Técnica de Ambato. Docente de la Carrera de Pedagogía de los Idiomas Nacionales y Extranjeros. wilmaesuarezm@uta.edu.ec
} 
del sexto y séptimo año de una escuela de educación primaria. Los resultados obtenidos se analizaron mediante la prueba estadística Chi-cuadrado, la misma que señala que el uso adecuado de los géneros literarios en actividades relacionadas con el desarrollo de la habilidad lectora del idioma inglés ha logrado grandes ventajas.

Palabras clave: Géneros literarios, lectura, inglés, grandes ventajas.

\section{Introducción}

Entre la literatura y el lenguaje existe una estrecha e innegable relación. La literatura se compone del lenguaje y esto proporciona ventajas para las aplicaciones del lenguaje. (Babaee \& Roselezam, 2014). La literatura se muestra como una herramienta valiosa en la enseñanza de las lenguas. Ciertamente, la literatura abre el camino para la enseñanza de lenguas y la adquisición de las mismas. Se ha considerado el empleo de la literatura como una valiosa e interesante estrategia por varios expertos de la enseñanza. (Sage, 2013). Es de suma importancia el uso de los géneros literario en el campo educativo. Este ayuda al desarrollo y desenvolvimiento del hombre influyendo positivamente en la adquisición de la lengua y a su vez en el desarrollo de sus destrezas. Las habilidades de escuchar, hablar, leer y escribir se conocen como destrezas del lenguaje. Ser competente en una lengua significa poseer estas cuatro habilidades.

Potencializar el uso de géneros literarios dentro del aula de clase de inglés crea viabilidad de interés lectora en el estudiante, logrando un aprendizaje adecuado del idioma. Y desde luego si de aprender un idioma se trata, leer ayuda mucho. Por lo que la utilización de las diferentes obras literarias en el proceso de aprendizaje del idioma inglés crea procesos de aprendizaje factibles y exitosos. El inadecuado uso de los géneros literarios provoca monotonía en el desarrollo de la lectura. A su vez, el inadecuado uso de los géneros literarios incita al desinterés en la lectura autónoma. Finalmente, esto también crea dificultad de interacción lectora.

Ante la necesidad de mejorar el proceso de aprendizaje del idioma inglés. Se llevó a cabo un trabajo de investigación para conocer el problema y dar posibles soluciones a esta necesidad. Con el propósito de mejorar la destreza de lectura del idioma inglés dentro de clases. El objetivo de esta investigación fue analizar las causas que afectan el desarrollo de la destreza de lectura del idioma inglés. Una vez analizado, se planteó señalar estrategias que sirvan para mejorar la lectura en inglés. Finalmente, exponer las ventajas del uso de los géneros literarios en la destreza de lectura, planteando una alternativa de solución al problema objeto de investigación.

\section{Marco Teórico}

\section{Géneros literarios}

De acuerdo a Aristóteles, los géneros son entidades poéticas de imitación que proceden como vínculo entre la realidad y la ficción literaria (Todorov, 2013). Adicionalmente, los géneros literarios son agrupaciones de obras literarias que emplean un lenguaje 
literario trasmitiendo sucesos reales debido a que poseen aspectos socio-culturales (Caiza, 2015). Los géneros literarios se instituyen en dos grandes autores; Aristóteles y Platón. Sin embargo, Aristóteles y Platón enfocan sus estudios con puntos de vista diferentes. Aristóteles enfoque su estudio de manera crítica, mientras que Platón basa su estudio de manera creativa. Una obra creativa tiene sensibilidad artística, es decir que crea un mundo de contexto imaginativo y su perspectiva al mundo alcanzan colores imaginativos con cierta sensibilidad artística, transportado al lector a mundos nuevos. Mientras que una obra critica discierne y juzga esa composición creativa. (Barad, 2012)

Varios autores conocedores del tema logran establecer solidas definiciones acerca de este asunto. Los géneros literarios son aquellos textos de discurso formal (Gómez, 2013). Asimismo, se explica que los géneros literarios son diferentes obras literarias con características esenciales comunes agrupadas entre sí (Frau,2002). Los géneros literarios juegan un papel primordial en la enseñanza del idioma inglés y aplicarlos correctamente dentro del aula alcanza grandes ventajas en el aprendizaje del idioma inglés. Los géneros literarios son excelentes materiales auténticos de enriquecimiento cultural, así como también materiales de beneficio y enriquecimiento de la lengua e intervención personal. Dejando claro que el uso de la literatura es un poderoso recurso en el contexto del aula para el aprendizaje de una lengua. (Collie \& Slater, 2010).

Ahora bien se puede determinar según lo establecido anteriormente que los géneros literarios son herramientas de carácter educativo que mejorar las habilidades y destrezas que el idioma inglés. El uso de los géneros literarios establece un aprendizaje correcto. Si no se diera énfasis en el uso adecuado de los géneros literarios en la destreza de lectura del idioma inglés dentro del salón de clases, obviamente la enseñanza se verá afectada. En el caso de no dar énfasis en el uso adecuado de los géneros literarios en la destreza de lectura del idioma inglés dentro del salón de clases, obviamente la enseñanza se verá afectada en el desarrollo de la destreza de lectura del idioma. Además, la enseñanza también se verá deteriorada en el aprendizaje de vocabulario y en el aprendizaje de estructuras gramaticales. Simultáneamente, la enseñanza se percibirá perjudicada en la monotonía de lectura, creando desmotivación al momento de aprender inglés. Ya que un aprendizaje correcto proviene del manejo y destreza de la lectura.

\section{Destreza lectora}

La calidad de la educación es el corazón del desarrollo. (Gove \& Cvelich, 2017). No cuestionable que la educación juega un papel importante para el progreso de la sociedad. Sin embargo, cada ecuatoriano lee un promedio de medio libro por año, mostrándose como la cifra más baja a nivel de Latinoamérica. (Andes, 2014). Consecuentemente, esto indica que es un reto para el docente es inducir al estudiante a la lectura ya que no se ha desarrollado un hábito de lectura.

La lectura es parte de la destreza receptiva del inglés y a su vez es una destreza esencial para los estudiantes de una lengua extranjera. El acto de leer es importante para un óptimo aprendizaje, ya que la lectura ayuda al estudiante a aprender a recordar vocabulario y 
estructuras gramaticales. Son varias las definiciones que se le otorga a la destreza de lectura. De acuerdo con Ruiz de Zarobe \& Ruiz de Zarobe (2011) la lectura es comprensiva caso contrario no es lectura. Asimismo, la destreza de lectura conlleva a procesos psicológicos y cognitivos, pues el lector emplea procesos de descifrado, así como la compresión del mismo.

Se denota leer como la acción de recoger, cosechar y adquirir. De modo que la lectura es el acto que da significado a un mensaje cifrado y al mismo tiempo crea en el lector puntos de vista personales. (Sastrías, 2014) Entonces, la destreza de lectura se la define entonces como la acción comprensiva del texto que conlleva al entendimiento y la interpretación de signos a través de los movimientos de los ojos. Este proceso activo encierra procesos; psicológicos y cognitivos, donde el lector comprende y da sentido al mensaje contenido en el texto por medio de la decodificación que es la capacidad de reconocer y procesar información escrita.

\section{Metodología}

La presente investigación muestra un enfoque cualitativo y cuantitativo, debido a que la finalidad de esta investigación es conocer una realidad contextualizada y examinar dichos datos estadísticamente. Por ende, plantea una modalidad bibliográfica, documental y no experimental ya que se desea conocer, comparar y deducir los enfoques, teorías y conceptualizaciones de diversos autores en el lugar en donde se produce los hechos de una realidad. Los niveles que se adoptaron como base de esta investigación son dos. El nivel exploratorio que pretende dar una visión general del estudio, permitiendo recolectar información trascendental de la situación del problema y de la hipótesis. Y el nivel descriptivo que no solo se limita a la recolección de datos, sino a la predicción e identificación de los vínculos que existen entre las variables. Además, presenta y resume la información de manera metódica. Y con ello se extrae generalizaciones significativas que contribuyan al conocimiento del desarrollo de la destreza de lectura del idioma inglés.

Se dio la participación de 48 estudiantes de sexto y séptimo año de la Escuela de Educación Básica Centro Escolar "Ecuador" del Cantón Ambato, Provincia de Tungurahua y de un docente del idioma inglés. Llevando a cabo encuestas estructuradas dirigidas a dicha población cuyo objetivo fue determinar la incidencia del uso adecuado de los géneros literarios en la destreza de lectura del idioma inglés. Según los datos recolectados a través de las encuestas realizadas se procedió a la realización de tablas y gráficos para dar lugar al análisis e interpretación de los mismo. Se aplicó la prueba estadística denominada chi cuadrado con el fin de aceptar la hipótesis inicial y rechazar la hipótesis nula y este a su vez dio lugar a la realización de conclusiones y recomendaciones del caso.

\section{Resultados}

Se analizó y se interpretó los resultados de la encuesta. Los resultados obtenidos de la encuesta dieron lugar a enfatizar las preguntas más relevantes de la misma. 
Tabla 1: Especificación de lo estadístico (Frecuencias Observadas)

\begin{tabular}{|c|c|c|c|c|}
\hline \multirow{2}{*}{ Preguntas } & \multicolumn{3}{|c|}{ Categorías } & \multirow{2}{*}{ 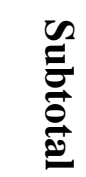 } \\
\hline & Opción 1 & Opción 2 & Opción 3 & \\
\hline Pregunta 3 & 5 & 12 & 31 & 48 \\
\hline Pregunta 4 & 9 & 29 & 10 & 48 \\
\hline Pregunta 6 & 5 & 9 & 34 & 48 \\
\hline Pregunta 8 & 4 & 7 & 37 & 48 \\
\hline \multirow[t]{2}{*}{ Pregunta 9} & 3 & 11 & 34 & 48 \\
\hline & 26 & 68 & 146 & 240 \\
\hline
\end{tabular}

Fuente: Encuesta dirigida a estudiantes

Elaborado por: Investigadores (2016)

Tabla 2: Frecuencias Esperadas

\begin{tabular}{llll}
\hline \multicolumn{1}{c}{ Preguntas } & \multicolumn{3}{c}{ Frecuencias Esperadas } \\
\cline { 2 - 4 } & Siempre & A veces & Nunca \\
\hline Pregunta 3 & 5,2 & 13,6 & 29,2 \\
Pregunta 4 & 5,2 & 13,6 & 29,2 \\
Pregunta 6 & 5,2 & 13,6 & 29,2 \\
Pregunta 8 & 5,2 & 13,6 & 29,2 \\
Pregunta 9 & 5,2 & 13,6 & 29,2 \\
\hline
\end{tabular}

Fuente: Encuesta dirigida a estudiantes

Elaborado por: Investigadores (2016)

Tabla 3: Cálculo de Chi-cuadrado

\begin{tabular}{ccc}
\hline Frec. Obs & Frec. Esp & \\
\hline 5 & 5,2 & 0,007692308 \\
9 & 5,2 & 2,776923077 \\
5 & 5,2 & 0,007692308 \\
4 & 5,2 & 0,276923077 \\
3 & 5,2 & 0,930769231 \\
12 & 13,6 & 0,188235294 \\
29 & 13,6 & 17,43823529 \\
\hline
\end{tabular}




\begin{tabular}{ccc}
\hline 9 & 13,6 & 1,555882353 \\
7 & 13,6 & 3,202941176 \\
11 & 13,6 & 0,497058824 \\
31 & 29,2 & 0,110958904 \\
10 & 29,2 & 12,62465753 \\
34 & 29,2 & 0,789041096 \\
37 & 29,2 & 2,083561644 \\
34 & 29,2 & 0,789041096 \\
& Chi 2 Calculado & 43,27961322 \\
\hline
\end{tabular}

Fuente: Encuesta dirigida a estudiantes

Elaborado por: Investigadores (2016)

\section{Gráfico 1: Curva estadística de aceptación y rechazo}

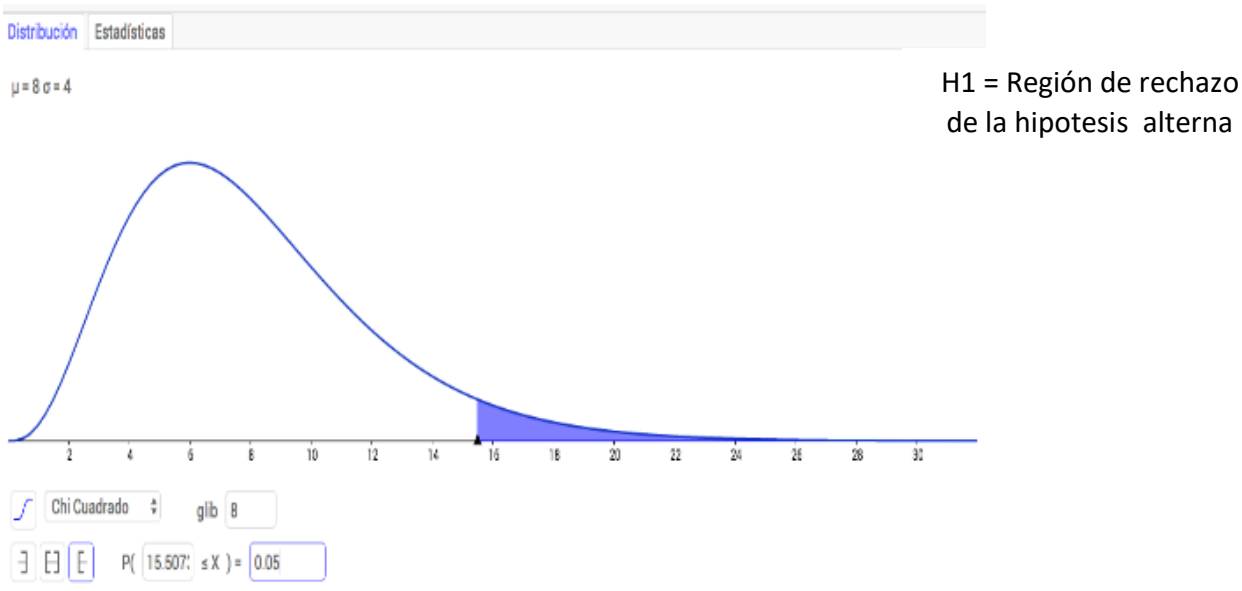

Fuente: International GeoGebra Institute. Cálculo Chi cuadrado.

Elaborado por: Investigadores (2016)

Con relación a los datos recolectados, es evidente que las lecturas de este aspecto misterioso o de personajes sobrenaturales no siempre son usadas en el aula para mejorar la destreza de lectura. Las lecturas que presentan situaciones de aspecto misterioso o de personajes sobrenaturales facilita el aprendizaje del uso figurado y cotidiano del lenguaje. Por lo que si no se aplica lecturas de este aspecto se debilita el desarrollo de la destreza de lectura en inglés. Una vez analizados los datos se puede establecer que no siempre se realiza lecturas en inglés que exhiban acontecimientos reales para mejorar la destreza de lectura del estudiante, amenorando el progreso de la habilidad lectora del idioma inglés. Se puede establecer según los datos recolectados que dentro del aula no siempre el estudiante utiliza poemas, cuentos, comics o bibliografías en inglés que le permitan la compresión del lenguaje y decodificación del significado de las palabras. Siendo una barrera para el progreso y desarrollo de la habilidad lectora del idioma inglés. 
De acuerdo a la información obtenida, se pudo determinar que las diferentes clases de palabras gramaticales (sustantivos, verbos, etc.) son raramente reconocidas en su mayoría por los estudiantes de sexto y séptimo año de la Escuela de Educación Básica Centro Escolar "Ecuador". Lo que no favorece al aprendizaje del idioma inglés tanto como en la compresión total lectora. De los datos recolectados se establece que la retención de fragmentos del texto en inglés no siempre es lograda por los estudiantes, por lo que el aprendizaje del idioma inglés se ve afectado.

\section{Análisis e Interpretación}

Dentro de las actividades del salón de clases es fundamental el uso de los géneros literarios. La literatura proporciona apoyo importante en la adqusición de un lenguaje debido a que ayuda a la memorización del estudiante, así como fuente de placer lector y transmisión de valores y conocimiento sin grado alguno de problema. (Jiménez, 2014). Al existir un limitado conocimiento de aplicación de actividades fundamentados en géneros literarios se genera monotonía en el desarrollo de la lectura. Así como también, desinterés total de lectura autónoma de las diferentes obras literarias. Y finalmente, dificultad de interacción lectora.

El uso adecuado de los géneros literarios brinda un progreso en la comprensión lectora. Además, ofrece ganancias léxicas apreciables. (Sacramento, 2015). Es evidente que, los géneros literarios juegan un papel primordial en la enseñanza del idioma inglés y aplicarlos correctamente dentro del aula alcanza grandes ventajas en el aprendizaje del idioma inglés. Los géneros literarios son excelentes materiales auténticos de enriquecimiento cultural, así como también materiales de beneficio y enriquecimiento de la lengua e intervención personal. Dejando claro que el uso de la literatura es un poderoso recurso en el contexto del aula para el aprendizaje de una lengua. (Collie \& Slater, 2010). Se recomienda avivar el desarrollo de las destrezas lectoras del idioma inglés mediante el uso de los géneros literarios, aplicando herramientas adecuadas para obtener así un impacto positivo en la adquisición del idioma inglés. La utilización de textos literarios brindan valores lingüísticos y socioculturales que ayudan a los estudiantes a desarrollar microhabilidades de las destrezas del idioma inglés (Caiza, 2015)

\section{Conclusión}

- El presente estudio exhibe en breve manera las ventajas del uso adecuado de los géneros literarios en la destreza de lectura del idioma inglés. Los géneros literarios exponen a los estudiantes a temas diversos e inesperados del lenguaje. Por otra parte, el uso de géneros literarios proporciona a los estudiantes el acceso a la cultura de la gente cuyo idioma ellos están estudiando. Además, el uso de las diferentes obras literarias puede ayudar a los estudiantes a ser más sensibles a características generales del inglés. (Sitman \& Lerner, 2015). Lo cual indica, que los docentes de idiomas pueden utilizar los géneros literarios en sus clases para de esta manera familiarizar a los estudiantes de un idioma con las particularidades 
básicas frecuentes del idioma inglés. Los estudiantes de un idioma, quienes está expuestos a los diferentes géneros literarios, mejoran su competencia lectora.

- De acuerdo a las indagaciones de investigaciones ya estudiadas se ve consolidada que el uso de los géneros literarios aporta al desarrollo de la destreza de escritura como lo menciona Caiza (2015), en su informe de investigación titulado " Los géneros literarios y la destreza de escritura del idioma inglés en los estudiantes del tercer año de Bachillerato General Unificado de la Unidad Educativa Temporal Jorge Álvarez del Cantón Píllaro", llegando a la conclusión que el uso de textos literarios fomenta micro habilidades en la destreza de escritura en los estudiantes debido a su valor lingüístico y sociocultural que aporta la literatura.

- Igualmente existe relación ante el estudio realizado por Andocilla, Albán y Tipanluisa (2015) con el tema: “Creación de Textos Literarios para fortalecer las Macro Destrezas, con los niños de Educación Inicial, del Centro Educativo Básico de Práctica Docente Semillitas "Cesar Francisco Naranjo Rumazo", en la parroquia La Matriz, cantón Pujili, provincia de Cotopaxi en el año lectivo 20142015" cuyo estudio presenta un enfoque cuantitativo, llevando a cabo como conclusión que las obras literarios son el fortalecimiento de las macro destrezas. Delimitando como realidad, que entre mayor sea el uso de los géneros literarios mayor será la oportunidad de mejorar las destrezas de lectura y de escritura del idioma inglés. Se manifiesta así que la integración de la literatura como actividad pedagógica dentro de clases genera impactos positivos en los estudiantes.

- Comparando el presente trabajo de investigación con el realizado por Jiménez (2015) quien como resultado manifestó que la literatura desde tiempos remotos aporta a la sociedad de conocimiento y valores. Es importante mencionar que los géneros literarios no solo proporcionan mejoramiento pedagógico en los estudiantes, sino que este a su vez también contribuye con valores a los estudiantes. Por otra parte, desde un enfoque diferente el presente trabajo de investigación con el elaborado por Jáimez (2015) en su estudio aprecia un mayor progreso en la expresión escrita del grupo de estudiantes mientras que la presente investigación estima mejoramiento en la destreza de lectura del grupo experimental, siendo esto un aspecto de diferencia en el estudio previamente realizado.

\section{Referencias Bibliográficas}

Andes. (23 de Abril de 2014). Día Mundial del Libro: Ecuador mantiene un bajo hábito de lectura. Ecuador: Agencia Pública de Noticias del Ecuador y Suramérica.

Andocilla Vega, M. B., Albán Yanchapanta, M. N., \& Tipanluisa Guilcaso, N. L. (Noviembre de 2015). Creación de textos literarios para fortalecer las macro destrezas, con los niños de educación inicial, del Centro Educativo Básico de práctica docente semillitas "Cesar Francisco Naranjo Rumazo", en la parroquia La Matriz, cantón Pujili, provincia de Coto. Latacunga, Cotopaxi, Ecuador. 
Babaee, R., \& Roselezam, W. (2014). Significado de la literatura en la enseñanza de lenguas extranjeras. Malaysia.

Barad, D. (2012). Literary Criticism.

Caiza Aucapiña, G. A. (2015). Los géneros literarios y la destreza de escritura del idioma inglés en los estudiantes del tercer año de Bachillerato General Unificado de la Unidad Educativa Temporal Jorge Alvarez del Cantón Píllaro. Ambato, Tungurahua, Ecuador.

Collie, J., \& Slater, S. (2010). Literature in the Language Classroom: A Resource Book of. Cambridge: CUP.

Frau, J. (2002). La teoría literaria de León Felipe. Sevilla: ISBN 8447206882.

Gómez Flores, A. M. (2013). MF1031_3: Expresión y Comunicación. IC .

Gove, A., \& Cvelich, P. (26 de Marzo de 2017). Early Reading: Igniting Education for All. Obtenido de http://www.uis.unesco.org/Education/Documents/early-readingreport_gove_cvelich.pdf

Jáimez Muñóz , S. (31 de marzo de 2015). El uso de textos literarios en la enseñanza de inglés en la educación secundaria. Andalucía, Granada, España.

Jiménez Ruiz, J. L. (2015). Iniciación a la lingüística. Club Universitario.

Jiménez Villacorta, P. (Noviembre de 2014). El uso de textos literarios en lengua inglesa en centros de educación primaria de Guadalajara. Guadalajara, México.

Rodríguez Guzmán, J. P. (2015). Gramática gráfica al juampedrino modo. Barcelona: Carena.

Ruiz de Zarobe, Y., \& Ruiz de Zarobe, L. (2011). La lectura en lengua extranjera. PORTALEDITIONS.

Sacramento, J. (2015). El uso de los textos literarios en la enseñanza del idioma inglés en la educación secundaria. Andalucía.

Sage, H. (2013). Incorporating Literature in ESL Instruction. New Jersey.

Sastrías, M. (2014). Caminos a la lectura. Pax México.

Todorov, T. (1988). Teoría de los Géneros Literarios. Madrid: Edic. Arco/ Libros S.A.

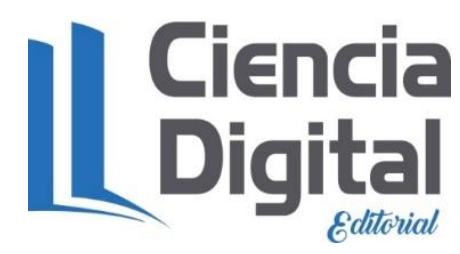


Para citar el artículo indexado.

Evelyn Vanessa, Vera de la Torre, A. J., Chimbo Cáceres, E. M., \& Suárez Mosquera, W. E. (2020). Géneros Literarios en la Destreza Lectora del Idioma Inglés. Explorador Digital, 4(2), 51-60. https://doi.org/10.33262/exploradordigital.v4i2.1200

\section{Ciencia Digital \\ Editorial}

El artículo que se publica es de exclusiva responsabilidad de los autores y no necesariamente reflejan el pensamiento de la Revista Explorador Digital.

El articulo queda en propiedad de la revista y, por tanto, su publicación parcial y/o total en otro medio tiene que ser autorizado por el director o editor de la Revista Explorador

Digital.
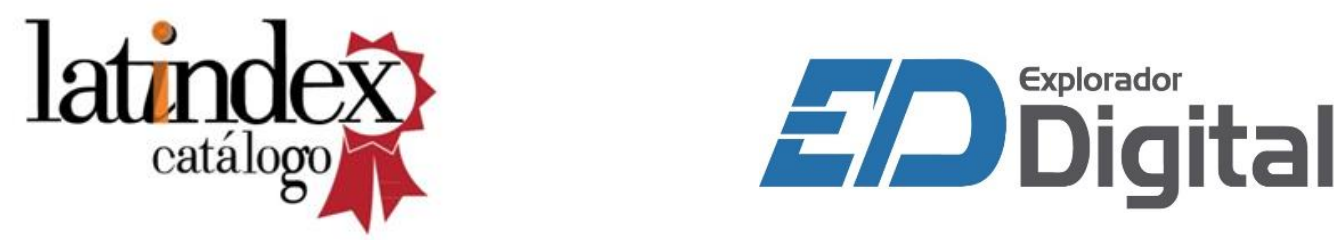